\title{
Change in patient nutritional knowledge following coronary artery bypass graft surgery
}

\author{
L. van Rooy", Yoga Coopoo \\ Department of Sport and Movement Studies, Faculty of Health Sciences, University of Johannesburg, 37 Nind Street, \\ Doornfontein Campus, 2092, Johannesburg, South Africa
}

\section{A R T I C L E I N F O}

Article history:

Received 15 September 2015

Accepted 9 January 2017

Keywords:

Coronary artery disease

CABG

Nutrition

Cardiac rehabilitation

Lifestyle modification

\begin{abstract}
A B S T R A C T
Introduction: In order to reduce coronary artery disease (CAD) risk, moderate physical activity should be combined with other lifestyle modifications, such as proper nutrition, to have a dramatic impact. This necessitates educational and preventative measures, which should begin in childhood and continue throughout life.

Aim: The aim of this study was to measure the change in nutrition knowledge of coronary artery bypass graft patients by implementation of a lifestyle intervention programme. Methods: The Hawkes and Nowak Nutrition Knowledge Questionnaire (1998) was administered to 18 coronary artery bypass graft (CABG) patients to assess the change in nutrition knowledge. Results: Significant improvements were noted in the nutrition knowledge score $(18.9 \pm 3.4$ $-23.2 \pm 4.5 ; \mathrm{p}=0.000)$. Although all components measured exhibited improvements in knowledge, cholesterol reduction knowledge $(5.3 \pm 1.8-7.2 \pm 1.8 ; \mathrm{p}=0.0066)$, low fat food knowledge $(3.8 \pm 2.3-5.1 \pm 2.7 ; \mathrm{p}=0.011)$ and high fibre food knowledge $(4.1 \pm 1.4-4.7 \pm 1.1$; $\mathrm{p}=0.022$ ) exhibited the highest and most significant improvements.

Conclusion: Notably, these significant improvements in nutrition knowledge points toward effective education being delivered during the intervention. Cardiac rehabilitation has proved to be effective in changing lifestyle habits in a holistic way and this study further shows an improvement in nutritional knowledge based on sound educational principles.

(C) 2017 The Authors. Publishing services by Elsevier B.V. on behalf of Johannesburg University. This is an open access article under the CC BY-NC-ND license (http:// creativecommons.org/licenses/by-nc-nd/4.0/).
\end{abstract}

\section{Introduction and background}

Cardiac rehabilitation can be defined as a comprehensive programme based on physical activity that represents multifaceted interventions aimed at improving prognosis by means of healthy lifestyle modifications. Programmes such as this, combined with nutrition, appropriate lifestyle modification and risk reduction play a major role in the management of cardiac conditions and have been shown to be highly beneficial in coronary artery bypass graft (CABG) patients as fundamental knowledge about lifestyle habits, such as nutrition, can contribute tremendously to an individual's level of wellness, including the enhancement of health and vitality (Durstine, Moore, Lamonte, \& Franklin, 2008; Jay, 2010; Lavie, Arena, \& Franklin, 2016; Robbins, Powers, \& Burgess, 2005). Studies conducted on Korean and Chinese university students

\footnotetext{
* Corresponding author.

E-mail address: lynnvr@uj.ac.za (L. van Rooy).
}

Peer review under responsibility of Johannesburg University. http://dx.doi.org/10.1016/j.hsag.2017.01.002

1025-9848/@ 2017 The Authors. Publishing services by Elsevier B.V. on behalf of Johannesburg University. This is an open access article under the CC BY-NC-ND license (http://creativecommons.org/licenses/by-nc-nd/4.0/). 
examined the relationship between nutrition knowledge and nutritional status, and have shown that good nutrition knowledge have positive effects on eating habits and food selection (Nti, Pecku, \& Opare-Obisaw, 2015). Despite substantial evidence and health knowledge pointing toward the protective nature that an adequate intake of fruit, vegetables and high fibre foods has against obesity, hypertension, cardiovascular disease, and diabetes South Africa still displays the classic signs of a population that is well established in nutrition-related non-communicable diseases (NCD's) (Abdulrahman \& Hazzaa, 2012; Buist, 1995; Shisana et al., 2013). With malnutrition being strongly associated with overweight and obesity, one factor in South Africa which has contributed to this pandemic is the frequency of meals being consumed outside of the home i.e. street foods, restaurant and fast food and take-away outlets (Ma et al., 2003; Shisana et al., 2013).The South African National Health and Nutrition Examination Survey (SANHANES-1) reported that 29\% of their sample (individuals of all ages living in South Africa, except those living in educational institutions, old-age homes, hospitals, homeless people and uniformed-service barracks) ate outside the home on a monthly basis, and $28 \%$ on a weekly basis (Shisana et al., 2013). These statistics further have a negative impact on cardiac risk, as well as morbidity and mortality, as it remains evident that many South Africans are consuming more foods which are rich in total fat and saturated fat, energy-dense, micronutrient-poor snacks and sweetened carbonated beverages, which are all insufficient to meet micronutrient needs (Vorster, Badham, \& Venter, 2013).

In a study conducted on women attending sport centres in Turkey, $17 \%$ of respondents stated that their preferred method of weight loss would be dietician-guided (Kozan \& Aktas, 2014). Although consulting a registered dietician is preferred regarding appropriate portion sizes and total calorie recommendations, improving nutrition knowledge through media and scientific literature may be beneficial regarding healthy and harmful foods and realistic ways to change bad eating habits. Faghri and Buden (2015) hypothesized that a negative relationship exists between nutrition knowledge and physical activity, and body mass index (BMI), implying that those with lower knowledge scores will have higher BMI values. Furthermore, increases in knowledge regarding nutrition may promote healthy weight loss behaviours (Laz, Rahman, Pohlmeier, \& Berenson, 2015). The transtheoretical model (TTM) of health behaviour change is a six stage model aimed at addressing these issues by interpreting behaviour change as a calculated process that unfolds over time (Prochaska, 2013). The underlying issues regarding behaviour change is the lack of comprehension regarding the health consequences of a particular behaviour. Research has indicated that many individuals find themselves in the first stage of TTM, the precontemplation stage, due to this lack of awareness (Prochaska, 2013). Although, nutrition knowledge alone may not be an adequate determinant of maintaining a healthy diet, knowledge can positively influence beliefs and facilitate healthier food intake practices (O'Brien \& Davies, 2007; Shisana et al., 2013). On the other hand, a study conducted by Pettigrew, Moore, Pratt, and Jongenelis (2015) on evaluation outcomes of a long-running adult nutrition education programme, concluded that the nutrition education programme improves the nutrition-related knowledge of participants as well as their behaviours.

At the outset, age, gender, genetics, occupation, lifestyle, family and cultural background have been acknowledged as factors that affect an individual's daily food choice (Shaw, 2004). An excessive dietary intake of fat and cholesterol has very strong and consistent epidemiological evidence supporting the association between nutrition and coronary artery disease (CAD) (Brubaker, Kaminsky, \& Whaley, 2002; Gordon \& Gibbons, 1991). Moreover, nutrition knowledge may also be influenced by cultural norms and beliefs regarding obesity; a problem affecting adults and children alike, as well as privileged and under-privileged communities (Puoane \& Tsolekile, 2008; Shisana et al., 2013). Although evidence suggests that disadvantaged individuals may be most vulnerable in terms of health knowledge since they do not have means of accessing health information, there is clear evidence that the majority of South Africans, regardless of their financial status and education level, have inadequate health knowledge (Shisana et al., 2013). For this reason, appropriate strategies aimed at improving health knowledge need to be directed towards the affluent and under-privileged communities' (Shisana et al., 2013).

\section{Problem statement and objective}

Cardiac rehabilitation has proved to be a very effective means of targeting health-related challenges in a holistic manner, since cardiac rehabilitation has evolved from prescribing simple exercises for the purpose of safe return to work or sport, to a multidisciplinary team approach incorporating a wide range of health care practitioners. Mampuya (2012) has emphasized that although the importance of primary prevention measures is aimed at delaying or preventing the onset of cardiovascular disease, cardiac rehabilitation mainly involves secondary prevention which relies on early detection of the disease process and the application of interventions such as education, counselling and behavioural strategies to further prevent the progression of disease, promote lifestyle change and modify risk factors. Clinical trials have proven that such strategies can retard, stabilize or even modestly reverse the progression of atherosclerosis and reduce cardiovascular events (Mampuya, 2012). Thus, the objective of this study was to measure the change in nutritional knowledge of CABG patients by implementation of a lifestyle intervention programme.

\section{Material and methods}

\subsection{Research design}

This study was a prospective intervention study. The questionnaire utilized was quantitative in nature, and explored the effects of exercise programmes, with particular emphasis on nutrition knowledge in CABG patients.

\subsection{Site of study and sampling}

The research was conducted at two private Biokinetic practices in Johannesburg as majority of Biokinetic practices in 
South Africa fall within the private sector. Furthermore, the sample was recruited from the private sector in order to delimit the study. A consent form was signed by each of the practice owners, granting their permission for utilization of these facilities. The purposive sampling method was used to recruit the convenience sample of 18 CABG patients $(n=17$ males; $n=1$ female) between the ages of 40 and 80 years. This cohort presented to the two respective practices during the period of February 2012-July 2014.

\subsection{Selection and recruitment of participants}

Permission to conduct research on participants was gained through ethical clearance, granted from the Academic Ethics Committee of the Faculty of Health Sciences at the University of Johannesburg.

Prior to commencement of this study, the researcher met with Cardiologists and Cardiothoracic Surgeons of the selected hospitals and explained the aims and objectives of the research. Cardiologists and/or Cardiothoracic Surgeons then referred patients to the study and those who met the inclusion criteria were invited to participate, after which an initial assessment was conducted. The inclusion criteria indicated that participants were required to be between the ages of 40 and 80 years, and should have no major chronic disease, excluding hypertension, diabetes, hypercholesterolaemia or a combination of two or three of the aforementioned health conditions. Participants with hypertension, diabetes, hypercholesterolaemia or a combination of two or three of these conditions were included into the study as these conditions are often found in patients who have had CABG surgery. Once a referral by a Cardiologist and/or Cardiothoracic Surgeon was made, the researcher visited prospective participants in the ward and explained the intended purpose, procedures, risks and benefits of participation in the study. Upon volunteering to take part in the study, informed consent forms were signed and the intervention stage commenced. Participants were aged between 40 and 80 years as research has shown that the mean age of myocardial revascularization, particularly CABG, was 63.4 years (Diodato \& Chedrawy, 2014).

The ethical principles of autonomy, beneficence, nonmaleficence, and justice were adhered to throughout the research process. Each participant was informed in writing of the procedures that were to take place, the purpose of the study, rights as a research participant, as well as any benefits and possible risks associated with the study, including muscle soreness, abnormal blood pressure responses, fainting, irregularities in heart rate, and heart attack. However, each participant was assured that every effort would be made to minimize such occurrences. Upon agreement of the disclosed implication of participation, participants finally signed an informed consent form, indicating their willingness to participate in the study.

\subsection{Measuring tools and instruments}

Each participant was initially assessed to determine specific needs and goals for rehabilitation and individualized exercise programmes were prescribed. The intervention phase of this study consisted of a twelve week lifestyle programme, which required participants to perform the prescribed exercise three times a week. During this time, each participant was issued with a lifestyle manual which outlined various nutritional guidelines related to risk reduction with respect to CAD. These nutritional intervention principles were based on broad categories to assist in behavioural changes, such as consumption of fruits, vegetables, cholesterol, protein, fibre, refined sugar, and total carbohydrates, as well as total fats broken down in its different components i.e., monounsaturated fats and polyunsaturated fats, and energy and/or calorie content per serving. A general introduction on how to interpret food labels was also included to create awareness and assist participants in improving food choices, hence encouraging healthier eating habits (Chopera, Chagwena, \& Mushonga, 2014). This included guidelines on identifying the order of ingredients on a food label and identifying whether the salt content in foods exceeded the daily recommendation. To supplement this, healthier alternatives to sugary and high fat foods, as well as which food types and habits to avoid, such as take-out meals, were also outlined.

The measuring tool used to assess nutritional knowledge pre- and post-rehabilitation, was developed by Hawkes and Nowak (1998a, 1998b). This Nutrition Knowledge Questionnaire is validated and was initially developed to assess the practical knowledge of cardiac patients and people at risk of heart disease (Hawkes \& Nowak, 1998a, 1998b). It was identified as an appropriate measurement tool for this study because its intended purpose and target population was similar to that of this research.

Ten checkbox type questions form the framework of this questionnaire and the total maximum score achievable is 38 points. The questionnaire was divided into subcategories, including food types avoided to reduce cholesterol (3 questions; 11 points), food types avoided to reduce weight (1 question; 9 points), foods low in fat content (4 questions; 11 points), foods high in fibre content (1 question; 6 points), and knowledge on food labels (1 question; 1 point). Although only one question on food label knowledge was asked in the questionnaire, it was mainly aimed at creating awareness and encouraging further reading in improving knowledge on food label analysis. Each subcategory was analysed, as well as the total score for the questionnaire, and the pre-test scores were compared to that of the post-test scores. Furthermore, permission was obtained from the authors to utilize the original copy of this questionnaire because it is a validated one, and the purpose for its original development was similar to that of this study. All exercise prescribed was supervised by the researcher, and weekly discussions took place regarding participants' nutritional behaviours and acquired knowledge.

\subsection{Data analysis}

The quantitative data collected from the nutrition knowledge questionnaire was analysed on a computer based programme called SPSS (Statistical Package for Social Sciences) for Windows version 15.0 (SPSS Inc., Chicago, IL, USA) by the University's Statistical Consulting Services. Descriptive statistics were computed and standard deviations were used in order to 
determine variance of the sample. All post-test data was compared to that of the pre-test data, in order to determine the effects of the intervention, and to what extent improvements in knowledge occurred.

The Kolmogorov-Smirnov and Shapiro-Wilk tests were done in order to determine normality. A nonparametric test, the Wilcoxon Signed Rank test, was used in order to determine whether there were significant differences between the data sets. This test was the most suitable for this set of data as the response variables were metric measurements with the presence of outliers (Lavange \& Koch, 2006). The Wilcoxon Signed Rank test was done to determine the significance over two time periods. For the Wilcoxon Signed Rank test, the level of significance was set at $5 \%(p \leq 0.05)$. Percentage change was included in the analysis of data, illustrating relative changes observed from pre-to post-test.

Effect sizes were also calculated as results are often better described in terms of measures of magnitude, such as how much a certain treatment will affect individuals (Sullivan \& Feinn, 2012). Cohen (1988) criteria indicates 0.1 to be a small effect, 0.3 a medium effect, and 0.5 is a large effect size.

\section{Results}

This study explored the changes in nutritional knowledge through education. In this section, nutritional data will be presented.

A total of 18 participants were invited to participate in the study with a mean age of 65.2 years. The youngest and oldest participants were 47 years old and 77 years old respectively. The mean height was $172 \mathrm{~cm}$ at the pre- and post-test and the shortest and tallest participants were $159 \mathrm{~cm}$ and $185 \mathrm{~cm}$, respectively. The mean weight increased slightly in the posttest by $0.3 \mathrm{~kg}(0.4 \%)$, thus also contributing to a $0.5 \%$ increase in mean BMI, with values ranging from 29.1 at the pre-test to 29.3 in the post-test, which both had a small effect size. A significant reduction of $1.9 \mathrm{~cm}(-2 \%)$ in mean waist circumference was observed from pre-test to post-test $(p=0.042)$, however, the smallest and largest waist circumferences remained the same at $83 \mathrm{~cm}$ and $117 \mathrm{~cm}$ respectively. Waist circumference reflected a medium effect size. Mean body fat percentage significantly reduced from $22.7 \%$ at the pre-test to $20.5 \%$ at the post-test, reflecting a $10 \%$ reduction $(p=0.000)$, and also had a large effect size.

Participants' knowledge regarding which food types to avoid to reduce cholesterol levels significantly increased from
5.3 to 7.2 out of 10 points ( $p=0.006$ ), comparing pre- and posttests. Similar results were observed concerning which foods should be avoided for weight reduction, with the score increasing again at the post-test (5.1-5.6 out of 9), however the increase was not significant and exhibited only a medium effect size. The category assessing knowledge of food types low in fat was out of 12 points and participants showed a significant increase in knowledge of $32 \%$ in the post-test ( $p=0.011$; large effect size). Knowledge regarding the types of foods that have a high fibre content also reflected a significant increase from 4.1 to 4.7 out of a total of 6 points $(p=0.022)$. Based on the nutritional guidelines participants received, the sample's food label knowledge, with particular emphasis on order of ingredients listed, showed a slight increase of $8 \%$, however, did not reflect significance and as a result displayed a small effect size. On the other hand, the mean total (out of 38 points) significantly increased from 18.9 in the pre-test to 23.2 in the post-test $(\mathrm{p}=0.000)$, reflecting a total nutrition knowledge increase of $23 \%$ as well as a large effect size.

\section{Discussion}

All participants resided in the Gauteng province in South Africa and had a mean age of 65 years.

When the results of the pre- and post-test questionnaire on nutrition are scanned, it is noted that significant changes had occurred in this cohort (Table 1). However, although the total knowledge score reflected a significant improvement and a large effect size, it may be perplexing that minor increases in weight and BMI had occurred. However, when noting the significant decreases in body fat percentage and waist circumference, this finding is not disappointing. The increases in BMI could be attributed to the increase in body weight, but the reduction in body fat percentage points toward a reduction in fat mass and an increase in lean mass (Shaw, Shaw \& Mamen, 2010). The possible explanation for this is that participants engaged in a strength training programme, which normally increases energy output. Moreover, aerobic training increases energy expenditure by activation of lipolysis and affects the reduction of body fat percentage (Chaudhary, 2010). Similarly, a study by Coopoo, Berger \& Andrews (1995) on a cohort of sedentary Indian males also showed decreases in body fat percentage after their intervention. While the findings regarding body weight in this study were not expected, it has been stated that exercise without weight loss

Table 1 - Demographic data of the sample $(n=18)$.

\begin{tabular}{|c|c|c|c|c|c|c|c|}
\hline & \multicolumn{2}{|c|}{ Pre } & \multicolumn{2}{|c|}{ Post } & \multirow[t]{2}{*}{$\%$ Change } & \multirow[t]{2}{*}{ Sig. } & \multirow[t]{2}{*}{ Effect size } \\
\hline & Mean \pm SD & Range & Mean \pm SD & Range & & & \\
\hline Age (yrs) & $65.2 \pm 8.0$ & $45-77$ & $65.2 \pm 8.0$ & $45-77$ & $0.0 \%$ & - & - \\
\hline Height (cm) & $172 \pm 8.3$ & $159-185$ & $172 \pm 8.3$ & $159-185$ & $0.0 \%$ & - & - \\
\hline Weight (kg) & $87.3 \pm 11.6$ & $63.7-107.5$ & $87.6 \pm 11.9$ & $63.4-107.5$ & $0.4 \%$ & 0.573 & Small \\
\hline BMI $\left(\mathrm{kg} / \mathrm{m}^{2}\right)$ & $29.1 \pm 3.7$ & $22.0-34.9$ & $29.3 \pm 3.6$ & $23.0-34.5$ & $0.5 \%$ & 0.397 & Small \\
\hline Waist circumference $(\mathrm{cm})$ & $99.7 \pm 8.3$ & $83-117$ & $97.8 \pm 8.8$ & $83-117$ & $-2 \%$ & $0.042^{*}$ & Medium \\
\hline Body fat percentage (\%) & $22.7 \pm 5.3$ & $14.0-33.7$ & $20.5 \pm 4.9$ & $13.0-31.0$ & $-10 \%$ & $0.000^{*}$ & Large \\
\hline
\end{tabular}


Table 2 - Change in knowledge regarding nutrition.

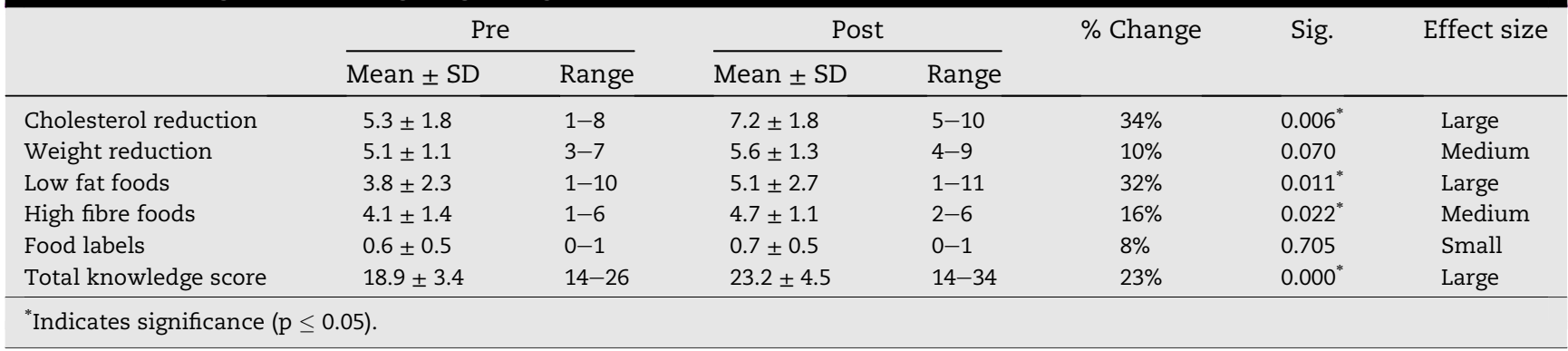

is associated with a substantial decrease in total and abdominal fat, hence a longitudinal twin study by Waller, Kaprio \& Kujala (2008) found that waist circumference was lower in active participants than inactive participants over a period of 30 years (Ross et al., 2004). These findings are in conjunction with the present study, as significant reductions in waist circumference were also observed among this group of participants (see Table 2).

Knowledge on cholesterol reduction and low fat foods exhibited the highest significant improvements, and the intervention largely affected both scores. This is noteworthy because diets in excess of fat and cholesterol are associated with CAD, and hence poor diet has been classified as a risk factor for heart disease (Brubaker et al., 2002; Gordon \& Gibbons, 1991; Myers, 2003). Research conducted on food quality and nutrition knowledge among Polish patients who had experienced a cardiovascular event found that $49 \%$ of men and $32 \%$ of women ate meat with visible fat (Waskiewicz et al., 2008). In a diet and exercise review on CABG patients, researchers found that total fat, saturated fat, and dietary cholesterol significantly decreased from the preoperative phase to the 1-year follow-up period (Coyan, Reeder, \& Vacek, 2014). Furthermore, it has been well established that lifestyle interventions, such as diet in combination with exercise, can be used to lower cholesterol levels in CABG patients (Coyan et al., 2014).

In research conducted by Laz et al. (2015), higher scores of nutrition knowledge was considerably associated with higher probabilities of engaging in healthy weight loss behaviours, such as eating smaller quantities, substituting high calorie foods to lower calorie foods, exercising, eating more fruits, vegetables and salads, and consuming less sugar.

Knowledge on high fibre foods also reflected a significant increase and yielded a medium effect size, which indicates that there is an equally divided chance that an intervention such as the one carried out in this study will have an impact on the knowledge of cardiac patients regarding foods which have a high fibre content. In a study highlighting the effects of dietary education on 200 patients following CABG surgery, it was found that $41 \%$ ate mixed-grain bread, $31 \%$ ate white bread, and only $28 \%$ ate brown bread, which points toward the necessity of forming new healthy eating patterns by providing education and nutritional counselling (Szczepariska, Brodzikowska, \& Calyniuk, 2014). Moreover, the more consumers know about nutrition, the more likely it is that they will understand and consult nutritional information on food labels (Soederberg Miller \& Cassady, 2015). In previous studies significant increases in patient understanding and motivation were found over the course of the study periods, which contributed to significant decreases in medication compliance rates for cardiovascular complications (Coyan et al., 2014). However, in this study a small effect size and non-significant result was found with knowledge related to food labelling. This could be attributed to the fact that only one food label question was assessed in the questionnaire and did not stimulate change in behaviour towards label reading.

\section{Conclusion}

The findings of this study have provided information regarding nutrition education. The intervention employed in this study showed improved knowledge regarding low-fat and high fibre food types, as well as those which reduce cholesterol levels, advocating that a twelve week programme with planned educational strategies is sufficient to obtain results regarding nutrition knowledge. In an attempt to rectify the lack of knowledge regarding nutrition amongst South African citizens, the Heart and Stroke Foundation of South Africa have recently implemented a "Salt Watch" campaign. This campaign, similar to the intervention regarding food label knowledge conducted in this study, may not necessarily change behaviours, but it is aimed at creating awareness regarding sodium intake and improving the ability to understand and read food labels, which would stimulate knowledge production. With this strategy in place, the Department of Health is a step closer in achieving its' aim of reducing mean population salt intake, proving that nutrition knowledge can successfully be improved (Byrne, Eksteen, \& Crickmore, 2014). In support of this initiative, health care practitioners should play a key role in patient education, since the public views primary health care providers as valuable sources of nutritional guidance to preventing and treating NCD's (Jackson, 2001; van Binsbergen, Delaney, \& van Weel, 2003; van Weel, 2003).

\section{Limitations}

A limitation of this study was the small sample size due the fact that recruitment only took place at private healthcare facilities. Additionally, participants were required to attend three supervised rehabilitation sessions a week, of which some found it challenging to commit to every appointment due to work commitments. Although they had indicated that 
they did exercise on their own, the researcher had no control over their exercise programmes in this instance.

\section{Recommendations}

The chief recommendation of this study is that cardiac rehabilitation encompassing nutrition knowledge and psychosocial guidance should be applied more strictly by medical personnel, both in the private and public sectors. This can be done at a fairly low cost and medical personnel should invest in further education to ensure that they are equipped with a sufficient body of knowledge that can be disseminated to cardiac patients.

Additionally, further research conducted on the CABG population should incorporate interventions from dieticians and clinical psychologists, in order to encourage the multidisciplinary team approach, which is the ideal setting for cardiac rehabilitation, as well as to improve the level of services rendered to the recovering CABG patient.

\section{R E F E R E N C E S}

Abdulrahman, O. M., \& Hazzaa, M. A. (2012). Prevalence and risk factors associated with nutrition-related non-communicable diseases in the Eastern Mediterranean region. International Journal of General Medicine, 5, 199-217.

van Binsbergen, J. J., Delaney, B. C., \& van Weel, C. (2003). Nutrition in primary care: Scope and relevance of output from the Cochrane collection. American Journal of Clinical Nutrition, 77(4), 1083S-1088S.

Brubaker, P. H., Kaminsky, L. A., \& Whaley, M. H. (2002). Coronary artery disease: Essentials of prevention and rehabilitative programs. Champaign, IL: Human Kinetics.

Buist, R. (1995). The cholesterol myth. South Africa: Struik Publishers.

Byrne, J., Eksteen, G., \& Crickmore, C. (2014). Heart and stroke foundation South Africa: Salt reference manual. South Africa: Heart and Stroke Foundation.

Chaudhary, S., Kang, M. K., \& Sandhu, J. S. (2010). The effects of aerobic versus resistance training on cardiovascular fitness in obese sedentary females. Asian Journal of Sports Medicine, 4, 177-184.

Chopera, P., Chagwena, D. T., \& Mushonga, N. G. T. (2014). Food label reading and understanding in parts of rural and urban Zimbabwe. African Health Sciences, 14(3), 576-584.

Cohen, J. (1988). Statistical power analysis for the behavioural sciences (2nd ed.). New Jersey: Lawrence Erlbaum.

Coopoo, Y., Berger, G. M. B., \& Andrews, B. C. (1995). The effects of an exercise and diet programme on coronary risk factors in a sedentary Indian cohort. African Journal for Physical, Health Education,. Recreation and Dance, 2, 80-88.

Coyan, G. N., Reeder, K. M., \& Vacek, J. L. (2014). Diet and exercise interventions following coronary artery bypass graft surgery: A review and call to action. The Physician and Sportsmedicine, 42(2), 119-129.

Diodato, M., \& Chedrawy, E. G. (2014). Coronary artery bypass graft surgery: The past, present, and future of myocardial revascularization. Surgery Research and Practice, 2014, 1-7.

Durstine, J. L., Moore, G. E., Lamonte, M. J., \& Franklin, B. A. (2008). Pollock's textbook of cardiovascular diseases and rehabilitation. Champaign, IL: Human Kinetics.

Faghri, P., \& Buden, J. (2015). Health behavior knowledge and selfefficacy as predictors of body weight. Journal of Nutritional Disorders \& Therapy, 5(3).
Gordon, N., \& Gibbons, L. (1991). The complete heart recovery guide. Cape Town: University Press.

Hawkes, A., \& Nowak, M. (1998a). A validated nutrition knowledge questionnaire for cardiac patients. Australian Journal of Nutrition and Dietetics, 55, 21-24.

Hawkes, A., \& Nowak, M. (1998b). Hawkes and Nowak nutrition knowledge questionnaire. Australia: Townsville.

Jackson, A. A. (2001). Human nutrition in medical practice: The training of doctors. Proceedings of the Nutrition Society, 60(20), 257-263.

Jay, N. (2010). Nutrition and cardiac rehabilitation heals cardiovascular disease: A review. Gujarat Medical Journal, 65(2), 71-78.

Kozan, D., \& Aktas, N. (2014). Eating habits, practices associated with weight loss and nutrition knowledge of women attending to sport centres. Journal of Nutrition Education and Behaviours, 46(4S), S163-S164.

Lavange, L. M., \& Koch, G. G. (2006). Statistical primer for cardiovascular research. Circulation, 114, 2528-2533.

Lavie, C. J., Arena, R., \& Franklin, B. A. (2016). Cardiac rehabilitation and healthy life-style interventions: Rectifying program deficiencies to improve patient outcomes. Journal of the American College of Cardiology, 67(1), 13-15.

Laz, T. H., Rahman, M., Pohlmeier, A. M., \& Berenson, A. B. (2015). Level of nutrition knowledge and its association with weight loss behaviours among low-income reproductive-age women. Journal of Community Health, 40(3), 542-548.

Ma, Y., Elizabeth, R., Bertone, E. R., Stanek, E. J., III, Reed, G. W., Hebert, J. R., et al. (2003). Association between eating patterns and obesity in a free living us adult population. American Journal of Epidemiology, 158(1), 85-92.

Mampuya, W. M. (2012). Cardiac rehabilitation past, present and future: An overview. Cardiovascular Diagnosis and Therapy, 2(1), $38-49$.

Myers, J. (2003). Exercise and cardiovascular health. Circulation, 107, e2-e5.

Nti, A., Pecku, E., \& Opare-Obisaw, C. (2015). Nutrition knowledge, meal patterns and nutritional status of energy drink users in a Ghanaian University Christina. Journal of Human Ecology, 49(1-2), 1-7.

O'Brien, G., \& Davies, M. (2007). Nutrition knowledge and body mass index. Health Education Research, 22(4), 571-575.

Pettigrew, S., Moore, S., Pratt, I. S., \& Jongenelis, M. (2015). Evaluation outcomes of a long-running adult nutrition education programme. Public Health Nutrition, 1-10.

Prochaska, J. O. (2013). Transtheoretical model of behaviour change. Encyclopaedia of Behavioural Medicine, 1997-2000.

Puoane, T., \& Tsolekile, L. (2008). Challenges faced by the urban black South Africans in the prevention of non-communicable diseases. Health Nutrition Problems of Indigenous Populations (Chapter 1). Studies of Tribes and Tribals, 2, 9-14.

Robbins, G., Powers, D., \& Burgess, S. (2005). A wellness way of life (6th ed.). New York: McGraw-Hill.

Ross, R., Jansen, I., Dawson, J., Kungl, A. M., Kuk, J. L., Wong, S. L., et al. (2004). Exercise-induced reduction in obesity and insulin resistance in women: a randomized controlled trial. Obesity Research, 12(5), 789-798.

Shaw, B. S. (2004). Response of coronary artery disease risk factors to three modes of training in sedentary males (Unpublished Doctoral Thesis). Rand Afrikaans University.

Shaw, B. S., Shaw, I., \& Mamen, A. (2010). Contrasting effects in body composition following endurance, resistance and concurrent endurance and resistance training. Journal of Sports Medicine and Physical Fitness, 2, 207-213.

Shisana, O., Labadarios, D., Rehle, T., Simbayi, L., Zuma, K., Dhansay, A., et al. (2013). South African national health and nutrition examination Survey (SANHANES-1). Cape Town: HSRC Press. 
Soederberg Miller, L. M., \& Cassady, D. L. (2015). The effects of nutrition knowledge on food label use. A review of literature. Appetite, 92, 207-216.

Sullivan, G. M., \& Feinn, R. (2012). Using effect size - or why the $p$ value is not enough. Journal of Graduate Medical Education, 4(3), $279-282$.

Szczepariska, E., Brodzikowska, M., \& Calyniuk, B. (2014). Dietary education among patients following coronary artery bypass graft surgery - a necessity or an unnecessary luxury? Polish Journal of Thoracic and Cardiovascular Surgery, 11(1), 12-16. Vorster, H. H., Badham, J. B., \& Venter, C. S. (2013). An introduction to the revised food-based dietary guidelines for South Africa. South African Journal of Clinical Nutrition, 26(3), S5-S12.
Waller, K., Kaprio, J., \& Kujala, U. M. (2008). Associations between long-term physical activity, waist circumference and weight gain: a 30 year longitudinal twin study. International Journal of Obesity, 32, 353-361.

Waskiewicz, A., Piotrowski, W., Sygnowska, E., Broda, G., Drygas, W., Zdrojewski, T., et al. (2008). Quality of nutrition and health knowledge in subjects with diagnosed cardiovascular diseases in the Polish population-National Multicentre Health Survey (WOBASZ). Polish Heart Journal, 66(5), 507-513.

van Weel, C. (2003). Dietary advice in family medicine. American Journal of Clinical Nutrition, 77(4), 1008S-1010S. 